\title{
Comparative analysis of transcriptional regulation of several cell types' migration in Drosophila embryo
}

\author{
Y.A. Galimova1, D.E. Maslov², E.D. Shvedkina², Y.A. Fedorova², A.S. Khruscheva ${ }^{2,3}$, \\ S.A. Fedorova ${ }^{2,3 *}$ \\ ${ }^{1}$ Institute of Molecular and Cellular Biology SB RAS, Novosibirsk, Russia \\ ${ }^{2}$ Novosibirsk State University, Novosibirsk, Russia \\ ${ }^{3}$ Institute of Cytology and Genetics SB RAS, Novosibirsk, Russia \\ *e-mail:fsveta@bionet.nsc.ru
}

Key words: transcription regulation, cell migration, embryo, Drosophila, GAF

Motivation and Aim: Molecular-genetic nature of cell migration is evolutionarily conservative and has high homology in different cell types, organs and tissues, and is similar in many aspects with the movement of the immune system cells and metastatic tumors. The Drosophila melanogaster embryo is a good model for studying the mechanisms of cell migration. First, primordial germline cells (PGC) start to migrate individually through midgut towards somatic gonadal precursor cells at stage 9 . Beginning from stage 10, caudal visceral mesoderm (CVM) cells move as a streaming collective. Hemocytes ( $\mathrm{HCs}$ ) initiate a posterior migration along the ventral midline at stage 11. In contrast to CVM, HCs appear to move more independently, as individuals. All migration types involve loose streams of cells that appear to be controlled in their movement.

Methods and Algorithms: Using the Flybase data, gene sets with tissue-specific expression at the 1-11 stages of embryogenesis were formed. Among these, we select genes exhibit specific expression, confirmed by in situ hybridization experiments from Y-K Bae and coauthors study [1], through the BDGP (Berkeley Drosophila Genome Project) database, or by our studies. Transcriptional regulation data were obtained from DroId (A comprehensive database of gene and protein interactions) database.

Results: In this work, we compared the transcriptional regulation of 3 migrating cell types from the Drosophila embryo: PGC, CVM (precursors of longitudinal muscles of the gut), and HCs (the Drosophila equivalent of blood cells). We found that among 24 genes, supported the PGC migration, expression of 23 genes is regulated by transcription factor GAF. Also, among 73 genes shared gene expression profiles in both migrating CVM cells and HCs [1], expression of 64 genes are regulated by transcription factor GAF. Moreover, we revealed, that PGC migration is affected in the mutants of Trl gene that encodes the Drosophila GAF, as well as in mutants of GAF targets shg, upd 2 and $t l l$. Conclusion: Comparison of transcriptional regulation of genes shared in common across different migrating cell types suggested that transcription factor GAF is the potential key regulator of cell migration in Drosophila embryo.

Acknowledgements: The research has been supported by RFBR No. 18-34-00321mol-a.

\section{References}

1. Bae Y.K., Macabenta F., Curtis H.L., Stathopoulos A. (2017) Comparative analysis of gene expression profiles for several migrating cell types identifies cell migration regulators. Mech Dev. 148:40-55. 